\title{
A Hands-On, Arduino-Based Approach to Develop Student Engineering Skills and Introduce Cybersecurity Concepts to K-12 Students
}

\section{Mr. Robert Shultz, Drexel University}

Robert Shultz is a 3rd year Biomedical Engineering PhD student, and a GK-12 fellow at Drexel University.

Mr. Daniel Edward Ueda, GRASP Laboratory, University of Pennsylvania

Daniel Ueda is the Associate Director for Education and Outreach at the GRASP Laboratory, University of Pennsylvania. He earned a BS in Mechanical Engineering from Rensselaer Polytechnic Institute and a MS in Teaching Mathematics from Pace University. Ueda has worked as a product design engineer for four years and as a teacher of math, physics, and engineering for eleven years. He was involved with the NSF GK-12 program at Drexel University for four years. Ueda has been awarded Delaware Valley Science Teacher of the Year (2013), Lindback Distinguished Teaching Award (2013), and Philadelphia Geek of the Year (2013).

Jessica S Ward, Drexel University (Eng. \& Eng. Tech.)

Jessica S Ward has over 9 years of Engineering Education experience and is currently the Director of Operations for the DragonsTeach program supported by the National Math and Science Initiative and UTeach Institute and serves as the National Science Foundation STEM GK-12 Program Manager at Drexel University.

Dr. Adam K Fontecchio, Drexel University (Eng. \& Eng. Tech.) 


\section{A Hands-On, Arduino-Based Approach to Develop Student Engineering Skills and Introduce Cybersecurity Concepts to K-12 Students (Work in Progress)}

\section{Abstract}

Without a doubt, today's generation is one driven by computer-based technologies. The vast majority of students uses computers and mobile devices on a daily basis and regularly posts on social media sites. As computer-related fields continue to grow, a future job market rich in technology-based careers seems inevitable. At the same time, as personal and sensitive data is increasingly stored online, the task of protecting this information represents another alreadyflourishing career field certain to grow in the coming years. While many students can efficiently operate computers and mobile devices, most of these same students view computers as a sort of "black box" system, with little understanding of the inner workings of a computer. Consequently, students frequently take for granted the security of the information they store or post online. To encourage students to pursue STEM career paths well represented in the job market, this paper will discuss a set of lessons developed for high school students to introduce basic computer science principles, as well as the concept of cybersecurity.

Our NSF-funded program (Graduate Stem Fellows in K-12 Education, or GK-12) pairs University graduate students with area high school teachers in an effort to encourage high school students to pursue careers in the STEM (Science, Technology, Math, and Engineering) fields. To accomplish this, curriculum is developed by the teacher and graduate student, and based around the 14 Engineering Grand Challenges set forth by the National Academy of Engineering. Through each lesson, students are introduced to applications of the skills they are learning in the classroom and given an opportunity to practice those skills in a real-life setting. The study discussed here is one set of lessons jointly developed by a GK-12 fellow and a high-school teacher.

Throughout the course of the year, students participate in a series of hands-on activities relating to computer technologies and cybersecurity, including case studies, small group projects, and class discussions. Students conduct surveys of their peers and construct infographic displays illustrating cybersecurity-related data, such as average password length. At the same time, students begin building basic circuits, and using Arduino microcontrollers accomplish simple tasks, such as causing a single LED light to blink at a defined rate. The year culminates in the design and creation of Arduino-based biomimetic devices for user identification, where students apply their new computer programming and circuitry skills to build fingerprint scanner devices. Student learning, as well as any changes in projected career paths, is assessed by pre- and postsurveys. The authors hypothesized that completion of the project would result in increased student interest in pursuing STEM careers, as well as knowledge regarding project-related material. This project could be applied in high school classrooms to teach students about computer programming and circuitry, and to encourage students to consider STEM and cybersecurity-related career paths.

II. Introduction/Background 
STEM fields (science, technology, engineering, and mathematics) represent some of the most rapidly growing job markets in the world. Despite this increase in jobs, however, only $16 \%$ of high school students are proficient in mathematics and interested in pursuing STEM careers. ${ }^{1}$ If this trend continues, the National Math and Science Initiative estimates that by 2018, the US may be short up to three million high-skilled workers. ${ }^{2}$

One example of a rapidly growing STEM field is cybersecurity. As more and more sensitive information is stored in online databases, ensuring the security of this information is of paramount importance. In fact, protecting credit card numbers, private communications, and other personal data has been named one of the National Academy of Engineering's 14 Engineering Grand Challenges. ${ }^{3}$ While the vast majority of high school students exhibit proficiency in operating computer-based technologies, very few understand the underlying principles by which these machines operate. Because computer-related fields such as cybersecurity appear intimidating or immensely difficult, students may lack the confidence necessary to consider pursuing careers in these fields.

Arduino microcontrollers are an excellent tool for introducing core principles of circuitry and programming. Starter kits provide students with the components and instructions necessary to design and wire basic circuits with ease, while examples of code that can be utilized to perform rudimentary tasks, such as lighting an LED bulb, are readily available.

In this study, we sought to convince high school students of the need for improved cybersecurity, and familiarize them with computer systems through a series of hands-on Arduino-based activities. All lessons took place in a $12^{\text {th }}$ grade International Baccalaureate (IB) Design Technology class. The study culminated in a design challenge in which students worked in groups and applied their collective circuitry and programming knowledge to wire and code Arduino-based biometric fingerprint scanning devices for user identification.

\section{Methods}

This study was implemented at a local high school, with $12^{\text {th }}$ grade students in the second year of a 2-year Design Technology course. Data collection was conducted via web-based surveys. The same survey was taken by each student at the beginning and end of the academic school year. All surveys were conducted anonymously by a third party affiliate (not the GK-12 program or the public school system). Survey questions used were previously developed by a team of grant administrators and industry professionals for use in all participating GK-12 classrooms.

Survey data was analyzed via McNemar's Test, a standard nonparametric statistical test for paired samples. Any survey response lacking a corresponding response at the other timepoint was excluded from the analysis. Significance was evaluated at $p<0.05$. Details of the procedure follow.

IIIa. Preliminary Modules- Setting the Scene 
To introduce general cybersecurity concepts to high school students, a series of lesson plans were devised and executed in the classroom. These lessons were intended to get students thinking about online security, while also revealing irresponsible or unsafe online activity common among the general population.

Through the first activity, we aimed to start a discussion about online identity. Students were each given an index card, and asked to anonymously record their most recent online activity on the card in the form of social networking activity, search engine requests, recently viewed videos, recently played music, etc. Cards were then shuffled and redistributed so that no student received his or her original card. Based on the information on their new cards, students were then prompted to design a custom advertising scheme aimed at the original author of the card. The lesson ended in a class discussion on the roles of analytics and statistics in modern advertising, as well as the need to protect and secure information stored about each consumer. Students were able to extend the ideas covered in the online identity lesson to all types of data stored online, including more sensitive personal information, such as credit card numbers and private email conversations.

Next, in order to show that unsafe computing habits were widespread, students worked together to devise survey questions dealing with cybersecurity. For example, some questions asked how frequently passwords to online accounts were changed, or how complex these passwords were. Students then distributed this survey to their peers, collected and compiled data, and presented their findings via infographic visual aids. As students saw how widespread unsafe habits were online, the need for improved cybersecurity became evident.

IIIb. Introducing the Arduino to the High School Classroom

An electronics company donated 4 Arduino starter kits and fingerprint scanner components, via educational sponsorship. Similar companies often offer educational sponsorships through which Arduino materials can be acquired for no or reduced cost. 2 additional starter kits and scanner components were purchased as well, bringing the total number of groups up to 6 . This allowed group sizes to be reduced to an average of 3 students per group.

The starter kits came with a set of 15 mini-lessons, each of which introduced a new component. Each mini-lesson included a detailed wiring map, as well as sample code. Working within their groups, students first completed the mini-lesson (such as blinking an LED bulb at a defined rate) and then were given a small challenge (for example, creating a circuit where 2 LEDs blink together or in succession). The challenges required students to dissect and manipulate the sample code, allowing them to better familiarize themselves with the underlying logic behind the code, as well as language-specific syntax.

\section{IIIc. Design Challenge- Creative Application of Acquired Skills}

The project culminated in a design challenge in which students were required to build their own biometric devices for user identification. Arduino boards and components, including a fingerprint scanning component, were provided to each group. Final designs had to be able to distinguish between fingerprints, and provide some output when the user's fingerprint was 
scanned. For example, if student A's fingerprint was stored as the target print, a green LED could light when student A's finger was scanned, while a red LED could light when any other student's finger was scanned.

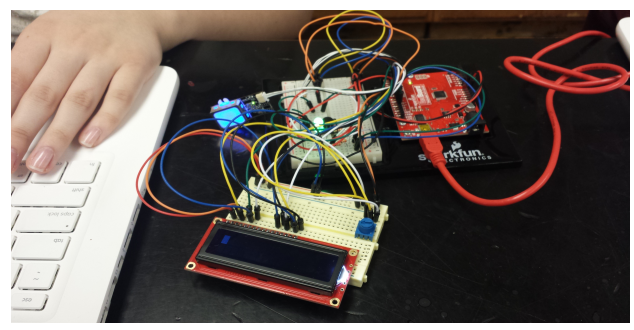

Figure 1. An example of a studentconstructed, biometric fingerprint scanning device for user identification. The scanning component is glowing blue in the provided image.

Students worked on their designs for several weeks. While not all groups were able to meet the design requirements, several designs could reproducibly distinguish between "user" and "hacker" fingerprint scans, and provide sufficient output to identify which fingerprint had been scanned. Additionally, if more time had been allotted for the design challenge, all groups would likely have been able to meet the full set of design requirements. Lastly, groups that did not achieve all of the design challenge goals still appeared to exhibit increased confidence and skill when faced with wiring and programming tasks.

\section{Results and Discussion}

Because this project was only conducted in a single classroom, data collection and analysis was limited by a small sample size $(n=13)$. Some data was collected via a set of surveys administered to students at the beginning and end of the academic year. Surveys asked students to report their top career choices.

\begin{tabular}{|l|c|c|}
\hline & Start of Year (\%) & End of Year (\%) \\
\hline & \multicolumn{2}{|c|}{ First Career Choice } \\
\hline STEM & 76.92 & 84.62 \\
\hline non-STEM & 23.08 & 15.38 \\
\hline & \multicolumn{2}{|c|}{ Second Career Choice } \\
\hline STEM & 61.54 & 61.54 \\
\hline non-STEM & 38.46 & 38.46 \\
\hline
\end{tabular}

Figure 2. Student career choices, reported as percentages; $\mathrm{p}>0.05$.

As shown in Figure 2, we found a modest 7.7\% increase in number of students interested in pursuing STEM careers as a first choice, and no change in the number of students interested in pursuing STEM careers as a second choice. This data was not, however, found to be statistically significant ( $\mathrm{p}>0.05, \mathrm{McNemar}$ 's Test).

A number of factors make this data difficult to interpret, however. Most students in an IB-level Design Technology course already have significant interest in STEM fields- this explains why our baseline percentage of students interested in pursuing STEM careers is so high above the 
national average. Additionally, this particular study was conducted in the second year of a twoyear Design Technology course, and most of the students had worked with a GK-12 fellow the year before. These factors also may have increased the baseline level of interest in STEM career paths in this particular classroom, making it difficult to determine overall efficacy of the project in terms of changes in projected student career paths. We suspect that a similar project conducted in a different classroom setting may yield more significant increases in STEM, and specifically computer-related career interests.

Finally, while changes in projected career paths were modest, we observed dramatic changes in student confidence and ability when working with the Arduino microcontrollers. For example, one particular student group initially exhibited frustration and voiced complaints when working through introductory Arduino mini-lessons. By the end of the mini-lessons, however, students in this same group where noticeably excited to get out the Arduino starter kits, worked throughout the entire period, and expressed a reluctance to put away the kits at the end of the class. This observational evidence illustrates how microcontroller-based projects can improve student confidence and ability in computer-related fields. Future work with similar projects in varied classroom settings would likely provide further insight.

\section{Conclusion}

In this study, we implemented a series of lesson plans designed to provide students with handson programming and circuitry, while simultaneously teaching students about the growing field of cybersecurity. This study makes a strong case for the use of Arduino-based lesson plans in the high school setting to teach cybersecurity concepts and generate interest in STEM and cybersecurity career paths. The authors found that the project discussed previously could be feasibly executed in the high school classroom, and yielded non-significant increases in STEM interest as assessed by pre and post-survey in a group of $12^{\text {th }}$ grade students already largely committed to pursuing careers in STEM-related fields. Despite this non-significant result, the authors feel that similar curricula could prove a very powerful tool, especially if implemented in a younger or less STEM-inclined classroom setting.

\section{References}

[1] (2015). Science, Technology, Engineering and Math: Education for Global Leadership. Available: http://www.ed.gov/stem

[2] (2015). STEM Education Statistics. Available: https://www.nms.org/AboutNMSI/TheSTEMCrisis/STEMEducationStatistics.aspx

[3] (2012). NAE Grand Challenges for Engineering. Available: $\mathrm{http} / / / \mathrm{www}$.engineeringchallenges.org/cms/challenges.aspx 